\title{
Transmission of Reverberant Sound Through Single Walls
}

\author{
By Albert London
}

\begin{abstract}
The transmission of reverberant sound through homogeneous single walls has been investigated theoretically and experimentally. Random incidence sound-transmission measurements were made on homogeneous walls of aluminum, plywood, and plasterboard. The results were found to be in satisfactory agreement with a modified version of a theoretical treatment first given by Cremer, which postulates that the wall impedance has a resistive component in addition to its mass reactance and a stiffness reactance resulting from the occurrence of flexural waves. Two of three parameters that are required to predict the transmission loss, namely, resistance, and critical flexure frequency, the third one being mass, are evaluated from the experimental data in such a way as to obtain the best fit between the theoretical and experimental results. Conditions under which the mass law of sound transmission may be assumed to be valid are investigated in detail by studying the behavior of Rayleigh's transmission law, which takes into account the compressional waves induced in the wall.

Experimentally, it was found that considerable improvement in transmission loss could be obtained by applying a fairly substantial sound-absorbent blanket to one of the faces of a homogeneous wall.
\end{abstract}

\section{Introduction}

Previous investigators $\left[\begin{array}{lll}1 & \text { to } & 5\end{array}\right]^{1}$ have given theoretical treatments for the attenuation of a plane sound wave upon transmission through multiple partitions consisting of two or more septa separated by airspaces. In all of these derivations only normally incident sound waves are considered. Consequently, it is difficult to compare the theoretical predictions with the experimental results obtained on walls for which transmission loss characteristics are determined [6] under the excitation of reverberant sound fields containing random waves with random angles of incidence.

Also in the previous derivations [ 1 to 5$]$, it has usually been assumed that each panel of the double wall combination has a mass reactance only. This is equivalent to assuming that each individual septum obeys the familiar mass law. However,

${ }^{1}$ Figures in brackets indicate the literature references at the end of this paper. it is well known that many homogeneous walls deviate considerably from the mass law. Consequently, it was necessary to investigate the transmission of reverberant sound through single walls. By utilizing a modified version of a treatment first given by Cremer [7], it was possible to determine a suitable equation of motion for each septum, which could then be inserted into the equations governing the transmission through the double wall.

In this paper, the attenuation of an obliquely incident plane sound wave upon transmission through a single wall is computed. By using the customary reverberant sound field statistics, the attenuation is integrated over all angles of incidence to give the average transmission loss. The same technique is applied in studying the transmission loss through double walls, which will be given in a subsequent paper. In addition to the theoretical treatment, experimental results on single and double walls will be given. 


\section{Transmission Through Single Walls}

\section{Attenuation of an Obliquely Incident Wave}

In figure 1 , an oblique plane wave is incident at an angle $\theta$ on the infinite wall. If $p_{i}$ is the acoustic pressure in the incident wave, then there will be a reflected wave with pressure $p_{r}$, and a transmitted wave with pressure $p_{i}$. It is required to determine the ratio $\mathrm{p}_{t} / p_{t}$. Consider any small area of the wall located at point $(0,0)$. Then

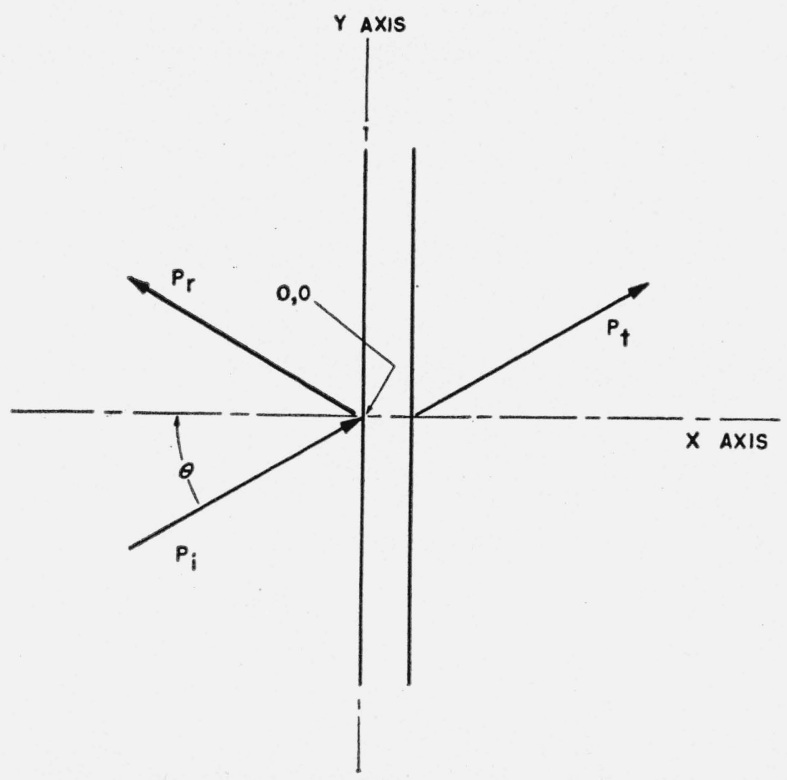

FIGURE 1. Geometrical relation between incident, reflected and transmitted pressure waves.

there are two conditions which obtain at the boundary between the sound field and the wall, which suffice to determine the desired ratio, that is, (1) continuity of $X$-component of particle velocity, and (2) the relation between the acoustic pressure difference acting across the interface and the motion of the wall.

Let the pressures be represented by expressions of the form:

$$
\left.\begin{array}{l}
p_{i}=P_{i} e^{i \omega t-i k(x \cos \theta+y \sin \theta),} \\
p_{\tau}=P_{\tau} e^{i \omega t-i k(-x \cos \theta+y \sin \theta),} \\
p_{t}=P_{t} e^{i \omega t-i k(x \cos \theta+y \sin \theta),}
\end{array}\right\}
$$

where

$$
k=\frac{2 \pi}{\lambda}=\frac{\omega}{c}, \lambda=\text { wavelength in air. }
$$

The directions of propagation of the waves are in the $x-y$ plane. As the particle velocity is related to the pressure by the expression

$$
\dot{\xi} x=\frac{i}{\rho \omega} \frac{\partial p}{\partial x}
$$

the first boundary condition at point $(0,0)$, results in the following:

$$
P_{i}-P_{r}=P_{t}=\frac{\rho c \dot{\eta}_{o}}{\cos \theta}
$$

where $\dot{\eta}_{0}$ is the velocity amplitude of the wall in the $x$-direction, whereas the second boundary condition requires that

$$
\left(P_{i}+P_{r}\right)-P_{t}=Z_{w} \dot{\eta}_{o}
$$

Here $Z_{w}$ is the mechanical impedance per unit area of the wall. Whence, $\alpha$, the ratio of the incident to transmitted amplitude becomes

$$
\alpha=\frac{P i}{P_{t}}=1+\frac{Z_{w} \cos \theta}{2 \rho c} .
$$

\section{Basic Assumptions}

In the derivation of eq. 1.6 it has been assumed that it is only necessary to consider a small area of the wall for which the projection of the wave front on it has practically constant phase. This treatment may be justified by the consideration that a reverberant sound field may be treated as the resultant of effects due to sections, having a small area, of wave packets with random angles of incidence and random phases. Also, use may be made of Schoch's [8] asymptotic law, which states that at sufficiently high frequencies above the fundamental frequency of a vibrating plate, the plate breaks up into small zones that vibrate independently of all other zones. Any small area of the plate vibrates in accordance with the sound pressure acting on this small area only.

Other assumptions that are implicit in this derivation can best be discussed with reference to a specific type of $Z_{w}$, that is, one in which the wall is assumed to have a mass reactance only, corresponding to which

$$
Z_{w}=i \omega m,
$$

where $m=$ mass of wall per unit area. Now, Rayleigh [9], by considering the compressional waves induced in the wall by the action of the 
sound waves, has shown that the attenuation is more generally given by

$\alpha=\cos \left(a_{1} l\right)+\frac{i}{2} \sin \left(a_{1} l\right)\left[\frac{\rho_{1} c_{1}}{\rho c} \frac{\cos \theta}{\cos \theta_{1}}+\frac{\rho c \cos \theta_{1}}{\rho_{1} c_{1} \cos \theta}\right]$,

where

$a_{1}=\frac{2 \pi}{\lambda_{1}} \cos \theta_{1}$.

$l=$ thickness of wall.

$\theta_{1}=$ angle of refraction of wave in wall.

$\rho_{1}=$ density of wall material.

$c_{1}=$ velocity of sound in wall material.

$\lambda_{1}=$ wavelength of sound in wall material.

In eq 2.2 a phase factor given by Rayleigh that does not effect the magnitude of $|\alpha|$ has been ignored since we shall concern ourselves only with absolute values. For $a_{1} l \ll 1$, or $2 \pi l / \lambda_{1}\left(\cos \theta_{1}\right) \ll 1$, eq 2.2 becomes

$$
\alpha=1+\frac{i}{2} \frac{2 \pi l}{\lambda_{1}} \cos \theta_{1}\left(\frac{\rho_{1} c_{1} \cos \theta}{\rho c \cos \theta_{1}}+\frac{\rho c \cos \theta_{1}}{\rho_{1} c_{1} \cos \theta}\right) .
$$

If we restrict ourselves to angles less than the critical angle as defined by Snell's law and as is explained below, then if $\left(\rho c \cos \theta_{1}\right) /\left(\rho_{1} c_{1} \cos \theta\right) \ll 1$, which is equivalent to $(\rho c) /\left(\rho_{1} c_{1}\right) \ll 1$ since $\cos \theta_{1}$ and $\cos \theta$ are of the same order of magnitude, eq 2.3 reduces to

$$
\alpha=1+\frac{i \omega m \cos \theta}{2 \rho c}
$$

which is identical with eq 1.6 when eq 2.1 is substituted in the latter equation. Thus, it will be seen that eq 2.4, which we shall call the mass law $\alpha$ is valid, provided the specific acoustic impedance of air, $\rho c$, is much less than that for the wall material, and the thickness of the wall is much less than the wavelength of sound in the wall.

It is of interest to investigate the behavior of eq 2.2 in the vicinity of $\theta=90^{\circ}$, since the mass law $\alpha$, (eq 2.4) predicts that the incident wave will be totally transmitted at grazing incidence. First, it is to be noted that, in accordance with Snell's law

$$
\frac{\sin \theta_{1}}{\sin \theta}=\frac{c_{1}}{c}
$$

and the critical angle of incidence $\theta_{c}$ is given by

$$
\sin \theta_{c}=\frac{c}{c_{1}}
$$

or since for ordinary materials $c_{1} / c \gg 1$, we see that the critical angle of incidence is rather small. Thus, for a brick wall $c_{1} / c \approx 10$, and therefore $\theta_{c} \approx 6^{\circ}$. For angles greater that $\theta_{c} \cos \theta_{1}$, which may be written as

$$
\cos \theta_{1}=\left\{1-\left(\frac{c_{1}}{c}\right)^{2} \sin ^{2} \theta\right\}^{1 / 2},
$$

becomes imaginary, i. e., $\sin \theta>c / c_{1}$. Then let

$$
\cos \theta_{1}=i\left\{\left(\frac{c_{1}}{c}\right)^{2} \sin ^{2} \theta-1\right\}^{1 / 2}=i \cos \theta_{1}^{\prime}
$$

where $\cos \theta_{1}^{\prime}$, from its definition, may be greater than unity, and also $a_{1}=i a_{1}^{\prime}=i\left(2 \pi / \lambda_{1}\right) \cos \theta_{1}^{\prime}$. Equation 2.2 becomes

$\alpha=\cosh \left(a_{1}^{\prime} l\right)-\frac{i}{2} \sinh \left(a_{1}^{\prime} l\right)\left[\frac{\rho c \cos \theta_{1}^{\prime}}{\rho_{1} c_{1} \cos \theta}-\frac{\rho_{1} c_{1} \cos \theta}{\rho c \cos \theta_{1}^{\prime}}\right]$.

If $a_{1}^{\prime} l \ll 1$, for $\theta>\theta_{c}$, then

$$
\frac{l \omega}{c_{1}} \cos \theta_{1}^{\prime} \approx \frac{l \omega}{c} \sin \theta \ll 1,
$$

so that this condition corresponds to $l \ll \lambda<\lambda_{1}$, and is to be compared with the condition $l \ll \lambda_{1}$ when $\theta<\theta_{c}$, previously derived. Equation 2.9 by a first-order expansion of $\cosh \left(a_{1}^{\prime} l\right)$ and $\sinh \left(a_{1}^{\prime} l\right)$ becomes

$$
\alpha=1+\frac{i}{2} \frac{m \omega}{\rho c} \cos \theta\left\{1-\left(\frac{\rho c}{\rho_{1} c_{1}}\right)^{2} \frac{\left[\left(\frac{c_{1}}{c}\right)^{2} \sin ^{2} \theta-1\right]}{\cos ^{2} \theta}\right\},
$$

which for angles close to $90^{\circ}$ reduces to

$$
\alpha=1+\frac{i}{2} \frac{m \omega \cos \theta}{\rho c}\left\{1-\left(\frac{\rho}{\rho_{1}} \tan \theta\right)^{2}\right\} .
$$

Thus total transmission occurs at an angle less than $90^{\circ}$ when $\tan \theta=\rho_{1} / \rho$, and since $\rho_{1} / \rho \approx 10^{3}, \theta$ is very close to $90^{\circ}$. At $90^{\circ}$, however, the attenuation becomes infinitely great. Furthermore for angles not close to $90^{\circ}$, eq 2.11 reduces to the mass law $\alpha$, (eq 2.4), even though $\theta>\theta_{c}$.

\section{Average Attenuation for a Reverberant Sound Field}

In a reverberant room, waves are incident at the transmitting wall from all possible directions. Since the phases are distributed at random, the 
energy of the resultant wave is a summation of the energy of each individual wave component. Each packet of energy incident at angle $\theta$, is greater than its corresponding transmitted packet by the factor $|\alpha|^{2}$. Let $\tau$ be the ratio of the total energy transmitted by the wall to the total energy incident on the wall, then in accordance with the usual reverberant sound field statistics [6]

$$
\tau=\frac{\int_{0}^{\frac{\pi}{2}} \frac{\cos \theta \sin \theta d \theta}{|\alpha|^{2}}}{\int_{0}^{\frac{\pi}{2}} \cos \theta \sin \theta d \theta}=2 \int_{0}^{\frac{\pi}{2}} \frac{\cos \theta \sin \theta d \theta}{|\alpha|^{2}} .
$$

For a wall having a pure mass reactance, i. e. $\alpha$ defined by eq 2.4, the transmission loss $(T L)$, which is defined as $10 \log _{10}(1 / \tau)$ is given by

$$
T L=10 \log \left(\frac{1}{\tau}\right)=10 \log a^{2}-10 \log \left[\ln \left(1+a^{2}\right)\right],
$$

where

$$
a=\frac{\omega m}{2 \rho c}
$$

Equation 3.2 is the random incidence mass law, which is to be distinguished from the normal incidence mass law given by

$$
T L=10 \log \left(1+a^{2}\right),
$$

which is readily obtained from eq. 1.6 and eq 2.1 by setting $\theta=0^{\circ}$ and noting that in this case $1 / \tau=|\alpha|^{2}$.

In table 1, a comparison between the normal and random incidence transmission loss values is made. Thus, it will be seen that the effect of random incidence is to materially reduce the transmission loss relative to what would be observed in the case of normal incidence.

Table 1. Transmission loss

\begin{tabular}{|c|c|c|}
\hline $\begin{array}{c}\text { Normal } \\
\text { incidence }\end{array}$ & $\begin{array}{c}\text { Random } \\
\text { incidence }\end{array}$ & Difference \\
\cline { 3 - 3 } & & \\
\cline { 3 - 3 }$d b$ & $d b$ & $d b$ \\
10 & 5.9 & 4.1 \\
20 & 13.3 & 6.7 \\
30 & 21.6 & 8.4 \\
40 & 30.4 & 9.6 \\
50 & 39.4 & 10.6 \\
60 & 48.6 & 11.4 \\
& & \\
\hline
\end{tabular}

Neither the normal nor the random incidence mass law agrees with experimental results on light- weight walls to be described below. However, it is possible to obtain reasonable agreement with experiment if it is postulated that the wall impedance $Z_{w}$ contains in addition to its mass reactance a resistive term. For this purpose it is assumed that

$$
Z_{w}=\frac{2 r}{\cos \theta}+i \omega m
$$

and in accordance with eq 1.6 , there results

$$
\alpha=1+R+i \frac{\omega m \cos \theta}{2 \rho c},
$$

where $R=r / \rho c$, the resistance in $\rho c$ units. Furthermore, in this case the transmission loss becomes

$$
T L=10 \log a^{2}-10 \log \left[\ln \left\{1+\left(\frac{a}{1+R}\right)^{2}\right\}\right] .
$$

From the method used in introducing the resistance coefficient $r$ in eq. 3.4, it will be seen that it has been assumed that a normally incident wave experience much less resistance than a wave at oblique incidence. This seems to be a natural assumption to make, since it is to be expected that a wave at almost grazing incidence would have high attenuation in passing through the material because of its long path length, whereas a normally incident wave would have little attenuation because of its short path length. In addition, from eq 3.5 it will be seen that the effect of the resistive term is to reduce the high transmission obtained at grazing incidence for the random incidence mass law. Since at low frequencies the mass reactance is rather small, while $R$ is independent of frequency, the effect of $R$ will be to increase the low frequency transmission loss, whereas at high frequencies it will have negligible effect.

In figure 2, application of the random incidence mass law, eq 3.2 , and the more general equation that includes the resistance term, eq 3.6, to experimental results obtained on a $1 / 64$-in. aluminum wall are shown. It will be seen that eq 3.6 may be made to fit the experimental data if $R$ is taken to be 2.16. The optimum value of $R$ was determined empirically so as to give the best fit with the data.

In the next section we shall consider modifications required in $Z_{w}$ when flexural waves are excited in the wall. Before taking leave of the 


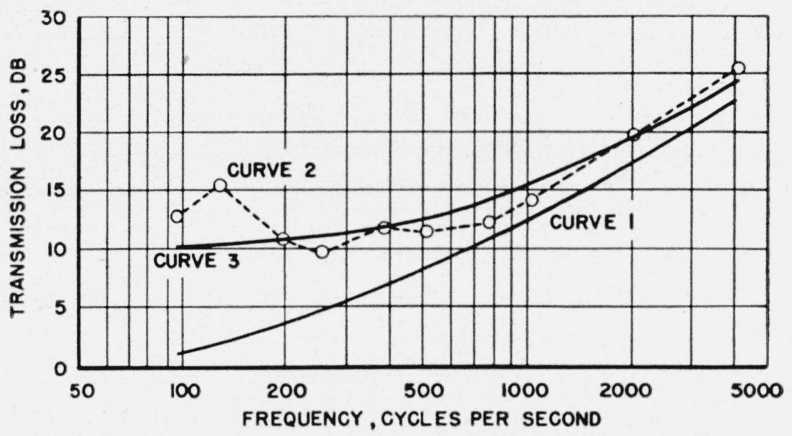

Figure 2. Transmission loss of a 1/64-in. aluminum wall.

$m=0.12 \mathrm{~g} / \mathrm{cm}^{2}$. Curve 1: transmission loss $=10 \log a^{2}-10 \log \left[\ln \left(1+a^{2}\right)\right]$; curve 2: experimental; curve 3 :

transmission loss $=10 \log a^{2}-10 \log \left[\ln \left\{1+\left(\frac{a}{1+R}\right)^{2}\right\}\right]$, where $R=2.16$.

present discussion we need to investigate further the relationship between Rayleigh's exact expressions (eq 2.2 and 2.9) and the random incidence mass law.

Since the mass law in the form of eq 2.11 holds only when $a_{l}^{\prime} l \ll 1$, it is of interest to determine what limitations occur as a result of this restriction. From eq 2.9, for $\rho c \cos \theta_{1}^{\prime} \ll \rho_{1} c_{1} \cos \theta$, which is valid for all values of $\theta$ except those very close to $90^{\circ}$, provided $\rho \ll \rho_{1}$, we may write

$|\alpha|^{2}=\cosh ^{2}\left(a_{1}^{\prime} l\right)+\frac{1}{4} \sinh ^{2}\left(a_{1}^{\prime} l\right)\left(\frac{\rho_{1} c_{1} \cos \theta}{\rho c \cos \theta_{1}^{\prime}}\right)^{2}$.

For small $a_{1}^{\prime} l$ we take $\cosh a_{1}^{\prime} l=1$ and $\sinh$ $a_{1}^{\prime} l=a_{1}^{\prime} l$. This substitution results in the mass law $|\alpha|^{2}$. Since both the cosh and sinh increase with increasing argument, we wish to find an upper bound to $a_{1}^{\prime} l$ such that the deviation of eq 3.7 from the mass law will not be too great. Furthermore, the second term of the right-hand side of eq 3.7 is much greater than the first, so that practically all of the deviation will be due to $\sinh \left(a_{1}^{\prime} l\right)>a_{1}^{\prime} l$. If we use the value of $a_{1}^{\prime} l$ such that $\sinh ^{2}\left(a_{1}^{\prime} l\right) /\left(a_{1}^{\prime} l\right)^{2}=2$, there will be approximately $3 \mathrm{db}$ more loss for this value of $a_{1}^{\prime} l$ as compared to the mass law. When $a_{1}^{\prime} l=1.5$, $\left[\sinh \left(a_{1}^{\prime} l\right)\right] /\left(a_{1}^{\prime} l\right)=1.4195$, which is a sufficiently close approximation to $\sqrt{2}$. This results in

$$
\sin \theta=\frac{1.5 c}{\omega l} ₹ 1
$$

where $\omega$ is $2 \pi$ times the frequency in $\mathrm{c} / \mathrm{s}, l$ is the thickness in centimeters. The value of $\theta$, provided $\theta \leqslant 90^{\circ}$, defined by eq 3.8 , say $\theta_{m}$, may be taken as the maximum angle of incidence for which the mass law expression (eq 2.4) represents very closely the Rayleigh expression (eq 2.9).

The whole state of affairs is pictured in figures 3 and 4 for the case of a brick wall for which the physical constants were taken as $\rho_{1}=1.5 \mathrm{~g} / \mathrm{cm}^{3}$, $c_{1}=4.3 \times 10^{5} \mathrm{~cm} / \mathrm{sec}, c_{1} / c=12.5, l=2$ in. for $f=$ $4,096 \mathrm{cps}$, or $l=4 \mathrm{in}$. for $f=2,048 \mathrm{cps}$, etc. It will be seen from figure 3 that $\theta_{m}$ in this case is about $24^{\circ}$, and the Rayleigh expression (eq 3.7) for angles greater than $\theta_{m}$ results in values for $|\alpha|^{2}$, which are much larger than the $|\alpha|^{2}$ obtained from the mass law. Figure 4 shows that the $\tau$ integral (eq 3.1), using the integrand $y_{m}$ corresponding to the mass law and integrating to $90^{\circ}$ will result in a value of $\tau$ that is much larger than that which the Rayleigh expression would give. To obtain an accurate value of $\tau$ under such circumstances, it would be necessary to integrate the exact Rayleigh expression. However, a rough approximation to the integral could be obtained by integrating the mass law expression from $\theta=0^{\circ}$ to $\theta=\theta_{m}$. The accuracy of this approximation would increase as $\theta_{m}$ increases. Thus, if we let $u=\cos ^{2} \theta$, and $\theta_{m}$ be the maximum angle
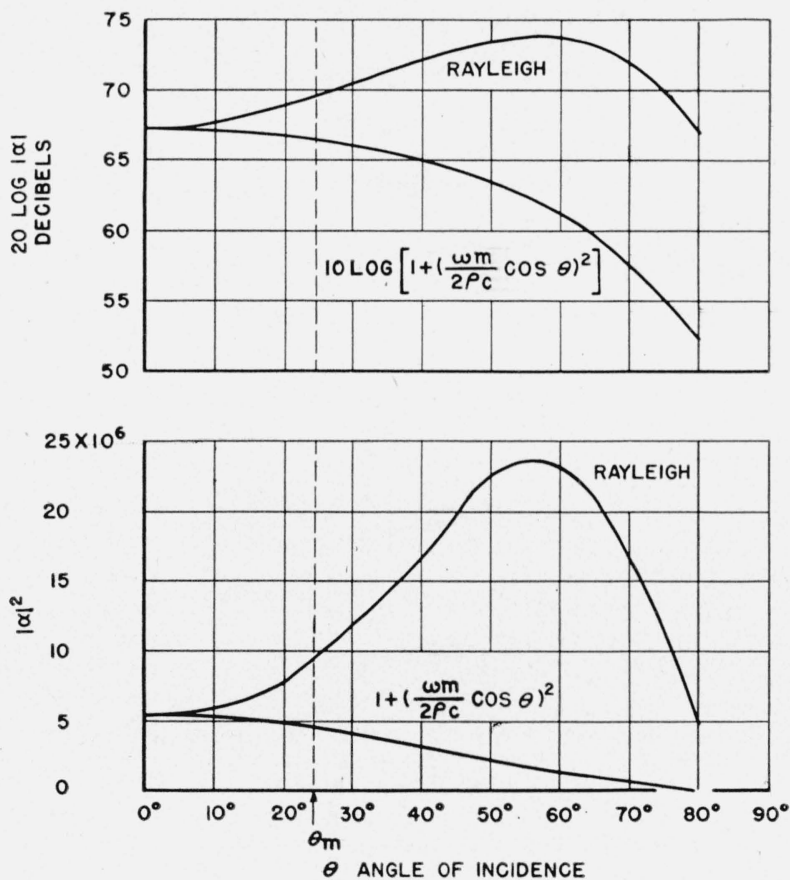

Figure 3. Comparison between $|\alpha|^{2}$ obtained from Rayleigh's expression, eq 2.9 and mass law $|\alpha|^{2}$.

Calculations for a brick wall: 2 in. thick at 4,096 c/s; 4 in. thick at 2,048 c/s; 8 in. thick at $1,024 \mathrm{c} / \mathrm{s}$, etc. 


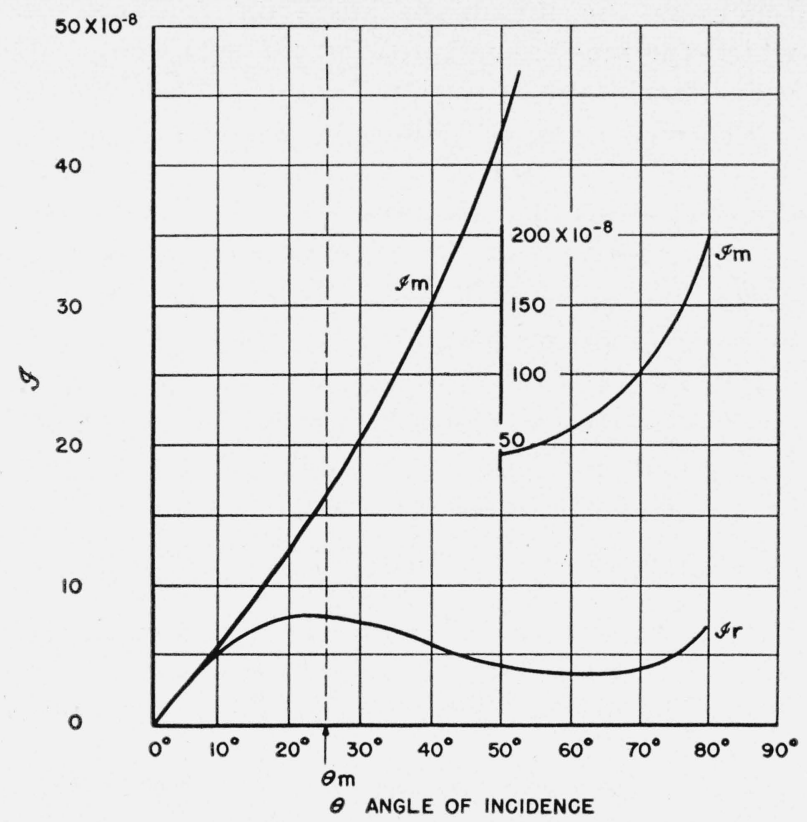

FIgURE 4. Comparison between $\tau$ integrand obtained from Rayleigh's expression, eq 2.9, and from mass law $|\alpha|^{2}$ (plotted in fig. 3 ).

$\mathscr{I}=\frac{2 \sin \theta \cos \theta}{|\alpha|^{2}}$,i. e., $\tau=\int_{0}^{\frac{\pi}{2}} \mathscr{I} d \theta ; \mathscr{I}$. corresponds to the Rayleigh expression $\mathscr{I} ; \mathscr{I}_{m}$ corresponds to the mass law $\mathscr{I}$.

of incidence for which the integral (eq 3.1) will be evaluated, we have from eq 3.8

$$
u_{m}=\cos ^{2} \theta_{m}=1-\frac{2.25 c^{2}}{\omega^{2} l^{2}},
$$

where $0 \leqslant u_{m} \leqslant 1$

and eq 3.1 becomes (using $Z_{20}=i \omega m$ )

Now

$$
\tau=\int_{u_{m}}^{1} \frac{d u}{1+a^{2} u}=\frac{1}{a^{2}} \ln \frac{1+a^{2}}{1+a^{2} u_{m}} .
$$

or

$$
a^{2} u_{m}=\frac{\omega^{2} \rho_{1}^{2} l^{2}}{4 \rho^{2} c^{2}}\left[1-\frac{2.25 c^{2}}{\omega^{2} l^{2}}\right]
$$

$$
u_{m}=1-\frac{0.56 \rho_{1}^{2}}{a^{2} \rho^{2}}
$$

Since $u_{m}=0$, when $\theta_{m}=90^{\circ}$, we see that restrictions on the maximum angle of integration begin to occur only when $0.56 \rho_{1}^{2} / a^{2} \rho^{2} \approx 1$, which, since $\left(\rho_{1} / \rho\right)^{2} \approx 10^{6}$, means that this phenomenon should be taken into account only when the wall is of such a nature that its normal incidence transmission loss is of the order of magnitude of $60 \mathrm{db}$ or greater. In this case it would be much better to use the exact Rayleigh expression than to attempt to use eq 3.10 .

\section{Flexural Waves; Experimental Results}

The case of flexural waves has been discussed in some detail by Cremer [7]. We shall state his results and point out wherein the present method differs somewhat from his presentation.

By using the wave equation for the motion of an infinite plate, Cremer shows that the wall impedance for a plane wave incident at any angle becomes

$$
Z_{w}=i \omega m\left[1-\frac{P \omega^{2}}{m c_{1}^{4}}\right]
$$

where

$$
\begin{aligned}
P & =\frac{Q l^{3}}{12\left(1-s^{2}\right)} \\
Q & =\text { Young's modulus } \\
s & =\text { Poisson's ratio } \\
c_{1} & =\text { Velocity of flexural wave. }
\end{aligned}
$$

In the above the velocity of the wall in the $x$-direction is represented by

$$
\dot{\eta}=\dot{\eta}_{0} e^{i \omega t-i k_{1} y},
$$

where $k_{1}=\omega / c_{1}=2 \pi / \lambda_{1}, \lambda_{1}=$ wavelength of sound in the wall. The wall thickness is assumed to be small compared to $\lambda$ and $\lambda_{1}$. Hence no $x$-dependent term occurs in $\dot{\eta}$.

The appearance of the negative reactance term in the wall impedance is evidently due to the plate stiffness. It will be seen that it is possible for $Z_{w}$ to become zero at the frequencies for which the bracketed term goes to zero in eq 4.1. The coupling between the acoustic wave and the flexural wave is given by the condition

$$
c=c_{1} \sin \theta,
$$

where $c$ is the velocity of sound in air. Furthermore, it is readily shown that if $c_{f}$ is the velocity of a flexural wave in the absence of driving forces, then

$$
c_{f}^{4}=\frac{P \omega^{2}}{m}
$$

so that $Z_{w}$ becomes

$$
Z_{v}=i \omega m\left(1-\frac{c_{f}^{4}}{c^{4}} \sin ^{4} \theta\right) .
$$


It is to be noted that $Z_{w}$ becomes zero when $c_{f}=c_{1}$, that is, when the velocity of the free flexural wave is the same as that of the driven flexural wave. This phenomenon Cremer has called the coincidence effect. The lowest frequency, $\mathrm{f}_{c}$, at which the coincidence effect takes place occurs when $\theta=\pi / 2$ and is given by

$$
\frac{P \omega_{c}^{2}}{m c^{4}}=1 \text {. }
$$

Now

$$
\frac{c_{f}^{4}}{c^{4}}=\frac{P}{m c^{4}} \omega^{2}=\frac{f^{2}}{f_{c}^{2}},
$$

or eq. 4.4 may be written as

$$
Z_{w}=i \omega m\left(1-\frac{f^{2}}{f_{c}^{2}} \sin ^{4} \theta\right) .
$$

By determining Young's Modulus from measurements on longitudinal waves in a long bar of the wall material, an expression for $P$ in eq 4.5 can be derived that results in the following expression for $f_{c}$

$$
f_{c}=\frac{\sqrt{3} c^{2}\left(1-s^{2}\right)^{\frac{1}{2}}}{\pi c_{l} l}
$$

where $c_{l}$ is the velocity of a longitudinal wave in the bar, and $l$ is the plate thickness.

So far we have not considered the effect of a resistance term in $Z_{w}$. Cremer introduces the dissipation effect by replacing $Q$ by $Q(1+i \epsilon)$, where $\epsilon Q$ is the complex part of Young's Modulus which results in damping. However, when it was attempted to use this method in experiments described below, extremely large values of $\epsilon$ were required to give any reasonable answers. In addition, the dissipation term had the wrong functional dependence on angle of incidence. It will also be seen that for frequencies much below $f_{c}$, eq 4.6 regains its simple mass law form, the complex Young's Modulus would have no effect, and this theory would predict no dissipation for thin partitions. Thus, for the $1 / 64$-in. aluminum wall shown in figure $2, f_{c}=30,000 \mathrm{cps}$, so that no flexural effects would appear at audio frequencies, and consequently no dissipation would appear if we followed Cremer's treatment. Accordingly, we introduce the resistance term here in the same way as in section II, 3 . The complete expression for $\alpha$, including flexural effects is then

$$
\alpha=1+R+\frac{i \omega m \cos \theta}{2 \rho c}\left(1-\frac{f^{2}}{f_{c}^{2}} \sin ^{4} \theta\right),
$$

and $\tau$ is obtained from $|\alpha|^{2}$ by eq 3.1. Unfortunately, the integral (eq 3.1) appears to be unintegrable in general in terms of elementary functions. Hence in what follows, numerical integration was utilized.

From eq 4.8 it will be seen that the transmission loss will depend on three constants $R, m$, and $f_{c}$. Of these three constants, $m$ is known, $f_{c}$ can be computed from eq 4.7 if all the other constants in this equation are known or measured, while $R$ is unknown. Experimental measurements of transmission loss were made on $1 / 2$-in. plywood, and $1 / 2^{-}, 1-$, and 2-in. plasterboard walls. The $R$ and $f_{c}$ were determined empirically by choosing those values that gave the best fit with experimental results. $R$ was determined by fitting an equation of the form eq 3.6 to the experimental data for frequencies from the lowest up to about one octave below the value of $f_{c}$.

In the case of figure 5, which is the transmission loss for a $1 / 2$-in. plywood wall, two critical flexure frequencies were computed, i. e., $f_{c}=900 \mathrm{c} / \mathrm{s}$, or $f_{c}=1,885 \mathrm{c} / \mathrm{s}$. The first value corresponds to $c_{b}$ for fir wood for a wave parallel to the wood fiber of $5.2 \times 10^{5} \mathrm{~cm} / \mathrm{s}$. The second value corresponds to $c_{l}$ for a wave perpendicular to the fibers equal to $2.4 \times 10^{5} \mathrm{~cm} / \mathrm{sec}$. The best fit was obtained for $f_{c}=1,885 \mathrm{c} / \mathrm{s}$. In addition to $f_{c}=900 \mathrm{c} / \mathrm{s}$, an $f_{c}=2,048 \mathrm{c} / \mathrm{s}$, where the $\operatorname{dip}$ in the experimental curve occurs, was also tried.

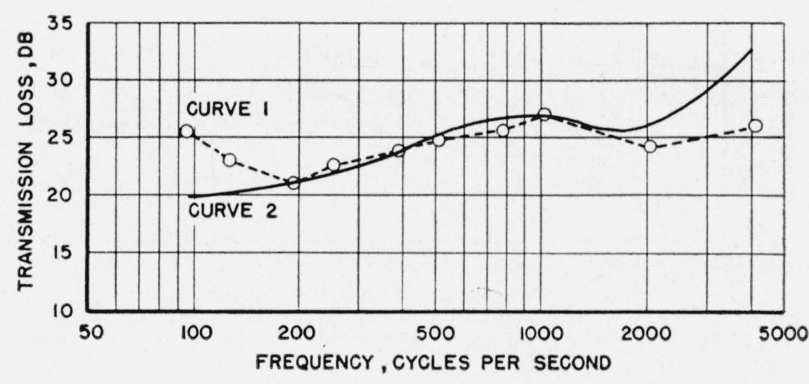

Figure 5. Transmission loss of a $1 / 2$-in. plywood wall. $m=0.70 \mathrm{~g} / \mathrm{cm}^{2}$. Curve 1: experimental; curve $2: R=8.3, \mathrm{f}_{\mathrm{c}}=1885$.

Figures 6, 7, and 8 give the results obtained on different thicknesses of plasterboard. In the $1 / 2$-in. plasterboard case, there is some doubt as to which is a better fit, $f_{c}=2,048 \mathrm{c} / \mathrm{s}$ or $f_{c}=4,096 \mathrm{c} / \mathrm{s}$. From eq 4.7 it is to be expected that $f_{c}$ for the various thicknesses of plasterboard should be inversely proportional to thickness. Consequently, if $f_{c}=512 \mathrm{c} / \mathrm{s}$ be taken as correct for the 2 -in. wall, 
then $f_{c}$ should be $1,024 \mathrm{c} / \mathrm{s}$ for the 1 -in. and 2,048 $\mathrm{c} / \mathrm{s}$ for the $1 / 2$-in. wall. The experimental results roughly approximate this sequence of values for $f_{c}$.

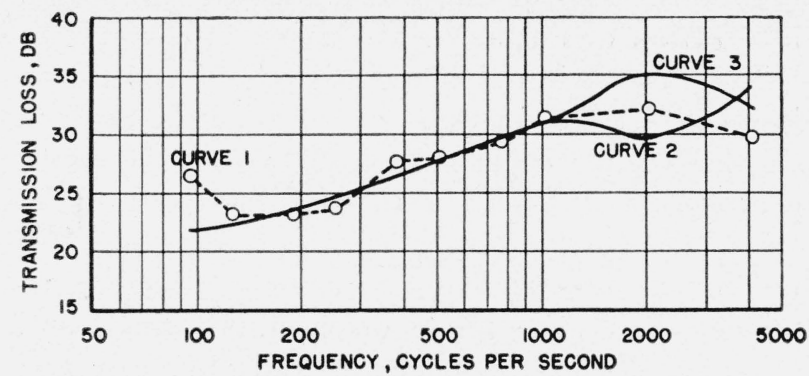

FIGURE 6. Transmission loss of a 1/2-in. plasterboard wall. $m=1.02 \mathrm{~g} / \mathrm{cm}^{2}$. Curve 1: experimental; curve $2: R=10.5, \mathrm{f}_{\mathrm{e}}=2,048$; curve 3 , $R=10.5, \mathrm{f}_{c}=4,096$.

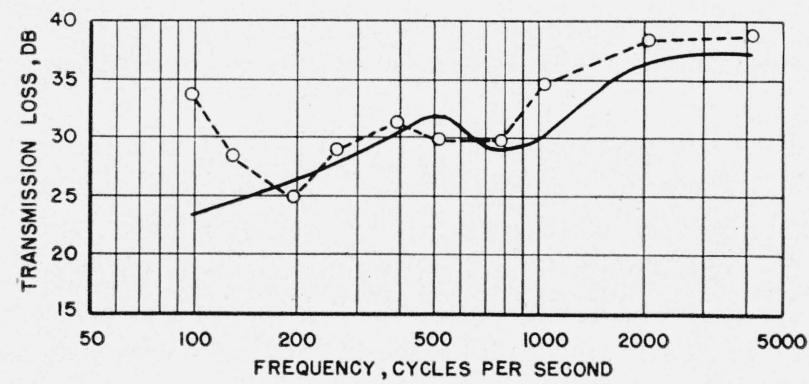

Figure 7. Transmission loss of a 1-in. plasterboard wall. $m=2.03 \mathrm{~g} / \mathrm{cm}^{2}$. Dotted curve: experimental; solid curve $2: R=10.5, f_{c}=768$.

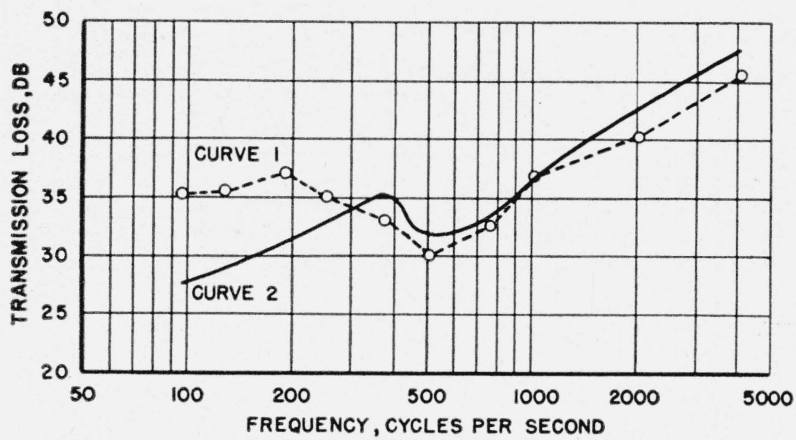

Figure 8. Transmission loss of a 2-in. plasterboard wall. $m=4.06 \mathrm{~g} / \mathrm{cm}^{2}$. Curve 1: experimental; curve $2, R=15.3, f_{c}=512$.

The variation of $R$ with thickness is worth noting. No appreciable change was observed between the $1 / 2$-in. and 1 -in. wall, whereas an approximately 50-percent increase occurred between the 1-in. and 2-in. wall. Although the experimental points lie above the theoretical curves at the lowest frequencies, it is not possible to change $R$ without changing the predicted transmission loss at the minimum point at which flexural effects are pronounced, and also the theoretical transmission loss curve in the lowfrequency region will go up or down accordingly as $R$ is increased or decreased. To some extent, the rather slow variation of $R$ with thickness indicates that some of the dissipation may be due to the type of mounting or restraints at the boundaries of the wall. Since this was the same for all three walls, it would not be expected to change. The walls were slightly less than 6 by $7 \frac{1}{2} \mathrm{ft}$ in size and were wedged in place in an opening of this size and then sealed in with a gypsum plaster seal. Experimental details are given in [6].

\section{Transmission Through Inhomogeneous Walls}

In this section we consider some additional experimental data bearing on the transmission of sound through single walls of an inhomogeneous character.

We consider the case of a wall having a thin veneer of a different material on its surface. The wall has a flexual frequency of $f_{c}$, occurring in the audio range, whereas the veneer has an $f_{c}$ outside of the audio range. It might be expected that such a combination would have an $f_{c}$ somewhere between the two extreme values, so that the composite panel would begin to deviate from its mass law characteristics at a frequency somewhat higher than that of the wall without the veneer, thus resulting in an over-all higher transmission loss. In figure 9 are shown the results on a composite panel of this type. In this experiment the base panel was the $1 / 2$-in. plywood wall of figure 5 , to which was applied a $1 / 64$-in. aluminum sheet on both faces by glue and tacks. No significant in-

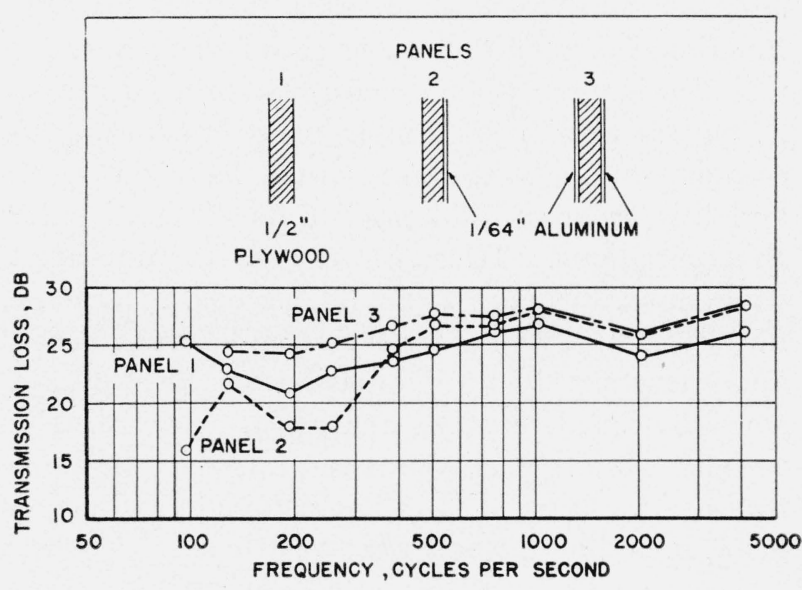

FIgURE 9. Transmission loss of a 1/2-in. plywood wall with $1 / 64$-in. aluminum faces. 
crease was observed over and above what would be expected on the basis of an increase in mass. Thus, it may be concluded that a thin veneer relative to a thicker core can have little effect on the over-all transmission loss. This bears on the question of the acoustic effectiveness of lining doors with metal.

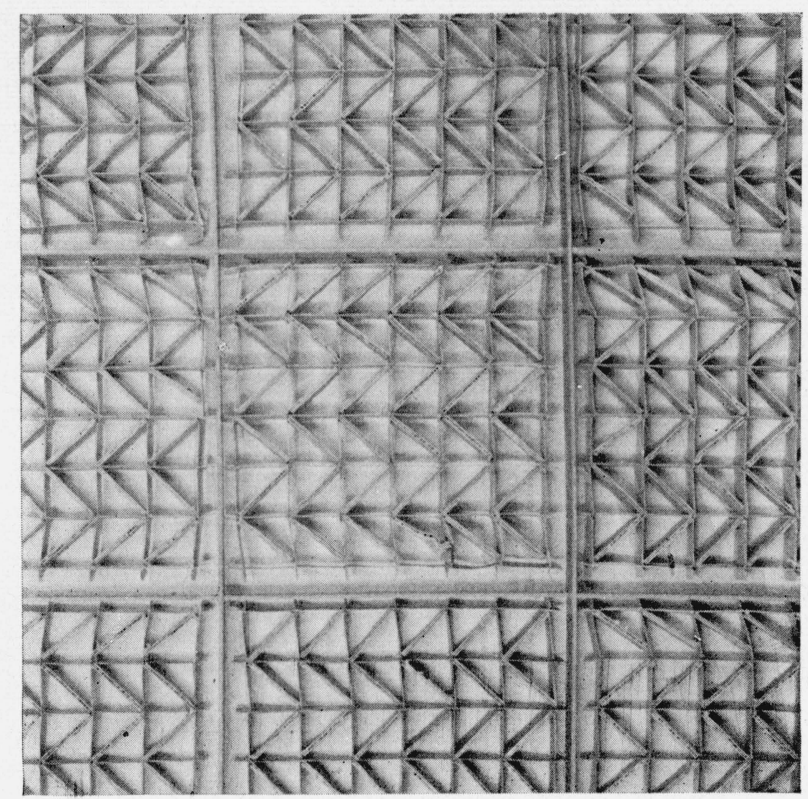

Figure 10. Egg-crate separators used to prevent transverse modes at wall surface.

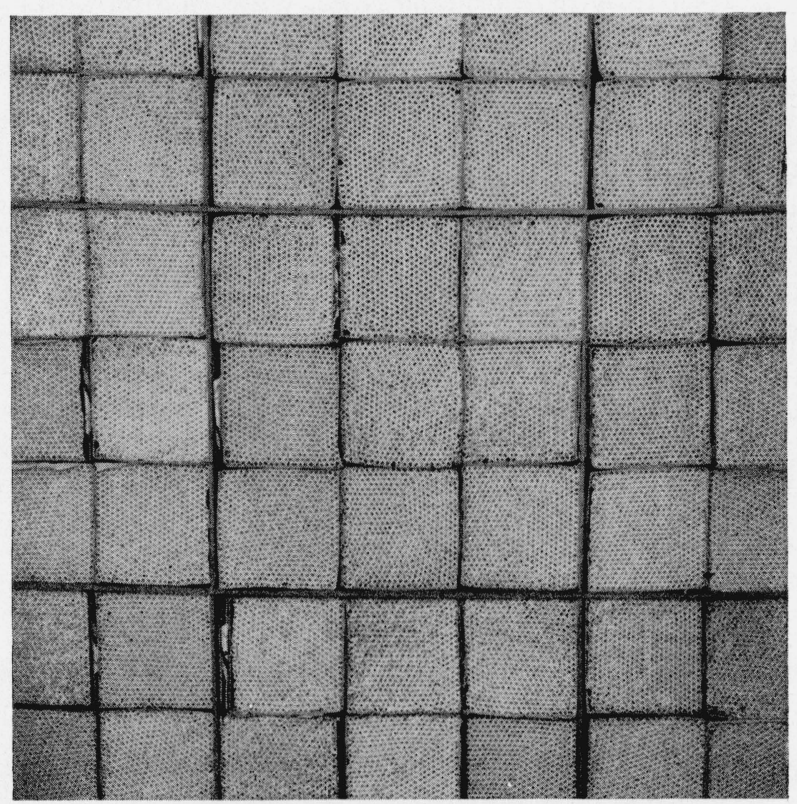

FIgURE 11. Strawcomb used in attempt to filter out waves incident at oblique angles.
Another type of experiment that was tried is illustrated in figures 10 and 11 . Here it was attempted to filter out obliquely incident waves by inserting a honeycomb structure in front of the wall so that waves travelling at or near grazing incidence would be impeded. The first structure tried consisted of egg crate separators shown in figure 10. The next structure consisted of soda straws about $2 \%$ in. long placed in front of the wall (fig. 11). There were approximately 150,000 straws used in the "strawcomb" shown in the figure. The principle involved is that if obliquely incident waves can be filtered out, then the transmission phenomena would be due solely to normally incident waves, thus eliminating flexural vibrations and raising the transmission loss compared to the random incidence case. The results obtained in a series of tests in which the strawcomb was placed in front of various thicknesses of plasterboard are shown in figure 12. Although some increase in transmission loss was experienced, it was hardly as much as one might expect. Upon considering the action of the straws in greater detail it will be seen that for frequencies such that the wavelength is much greater than the
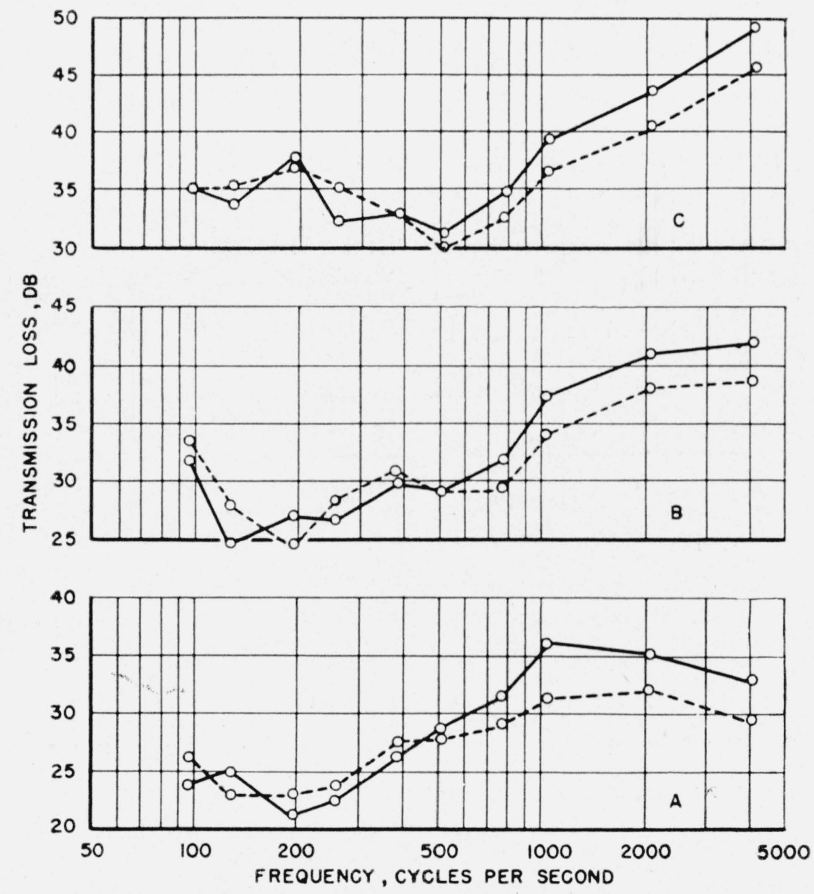

FIgure 12. Effect of placing strawcomb of figure 11 in front of plasterboard walls.

A, 1/2-in. plasterboard; B, 1-in. plasterboard; C, 2-in. plasterboard. tests made with $27 \%$-in. strawcomb in front of wall; - - strawcomb removed. 
$27 / 8$-in. straw length, the straws do not essentially change the boundary conditions (1) and (2) given in section II, 1. Thus the total pressure acting at the mouth of each straw is $p_{i}+p_{r}$, whereas the $x$-component of particle velocity is certainly continuous, inasmuch as this is the only component that would be transmitted down the straw. The particle velocity is increased somewhat inside the straw because the solidity, i. e., the ratio of wall volume to total volume, was of the order of 10 percent. We are thus led to the conclusion that a strawcomb of this type could not change the behavior of the wall to any great extent with the exception that transverse interference patterns or normal modes in the room located at the wall face would be broken up by the strawcomb. Meyer [2,3] has indicated that in the case of double walls such modes tend to reduce the effectiveness of the double wall. We shall discuss this use of the strawcomb in double walls in a subsequent paper.

The improvement in transmission loss obtained at the higher frequencies by use of the strawcomb may have been due to an absorbent effect of such a structure. Some measurements of the improvement in transmission loss obtained by hanging an absorbent blanket immediately in front on the source side of the plasterboard walls of figure 12 (without strawcomb) are shown in figure 13,

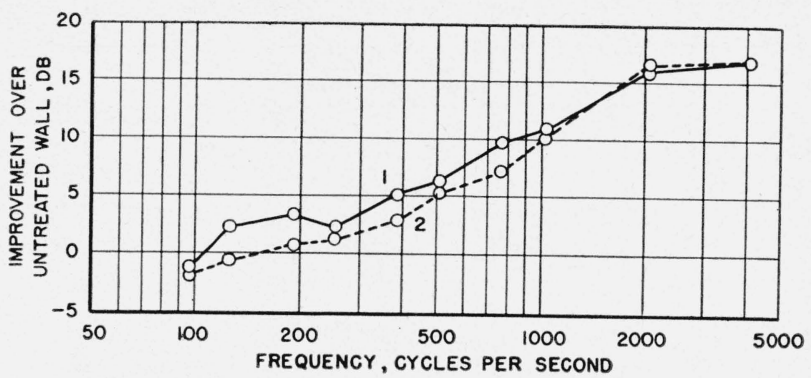

FIGURE 13. Effect of applying an absorbing blanket to a homogeneous wall.

--- --- 3-in. glasswool, $1.0 \mathrm{lb} / \mathrm{ft}^{2}$ on plasterboard walls; $13 / 4$-in. hairfelt, $1.2 \mathrm{lb} / \mathrm{ft}^{2}$ on plywood walls.

curve 1. The absorbent in this case was 3 in. of glass wool having an average density of $1 \mathrm{lb} / \mathrm{ft}^{2}$. The improvement resulting from the addition of the glass wool blanket was approximately the same for the $1 / 2-, 1-$, and 2 -in walls. Accordingly, the average increase in loss for these three walls is plotted in figure 13 . Curve 2 of figure 13 gives the results obtained on the improvement resulting from gluing on to the far or receiving room side of a $1 / 2-i n$. plywood wall, $1 \frac{3 / 4}{4}$ in. hair felt having an average density of $1.2 \mathrm{lb} / \mathrm{ft}^{2}$. Some additional improvement, of the order of $3 \mathrm{db}$, is obtained by gluing rather than hanging the blanket loosely. A general discussion of this problem has been given by Cook [10]. It will be seen from figure 13 that this type of treatment produces a rather large effect. Consequently, it should be borne in mind in practical noise reduction problems. In addition to the improvement in transmission loss, use may be made of the absorption provided by the blanket to increase the over-all noise reduction.

One other observation is probably pertinent from a practical point of view. In the case of the plasterboard wall series, if the improvement in transmission loss that results from doubling the mass of the wall is averaged over all 10 test frequencies, it will be found that doubling the mass results in an average improvement of approximately $4 \mathrm{db}$. This result is in accord with previous data $[11,12]$.

\section{Conclusion}

The experimental results and theoretical treatment show that there are three important physical properties of a homogeneous wall, namely, its mass, internal damping or dissipation, and its ability to propagate flexural waves, which determine its sound transmission loss. Furthermore, the importance of the effect caused by random angles of incidence of the sound waves in a reverberant room has been demonstrated. The experimental results were utilized to determine the values of $R$ and $f_{c}$ for the different walls in question. This effectively determines the value of the wall impedance, $Z_{w}$. Knowing $Z_{w}$ it will be possible to investigate the performance of double walls consisting of two single walls separated by an airspace. This will be considered in a subsequent paper.

The author acknowledges the assistance of Seymour Edelman and Henry J. Leinbach, Jr., who carried out the experimental observations. 


\section{References}

[1] J. E. R. Constable, Phil. Mag. 18, 321 (1934).

[2] E. Meyer, Elek. Nachr. Tech. 12, 393 (1935).

[3] E. Meyer, Z. Tech. Phys. 16, 565 (1935).

[4] D. G. Hunt, Can. J. Research 12, 398 (1935).

[5] A. L. Kimball, J. Acous. Soc. Am. 7, 222 (1936).

[6] A. London, J. Research NBS 26, 419 (1941) RP1388.

[7] L. Cremer, Akust. Z. 7, 81 (1942).

[8] A. Schoch, Akust. Z. 2, 113 (1937).

[9] Lord Rayleigh, Theory of sound 2, sec. 271, p. 186 (Dover, N. Y., 1945).
[10] R. K. Cook, Radiation of sound by a vibrating absorber, J. Acous. Soc. Am. 19, Abstract No. 35, p. 729 (1947).

[11] V. L. Chrisler and W. F. Snyder, Soundproofing of airplane cabins, BS J. Research 2, 897 (1929) RP63.

[12] Albert London, Principles, practice, and progress of noise reduction in airplanes, Tech. Note No. 748, National Advisory Committee for Aeronautics (Jan. 1940).

Washington, February 16, 1949. 